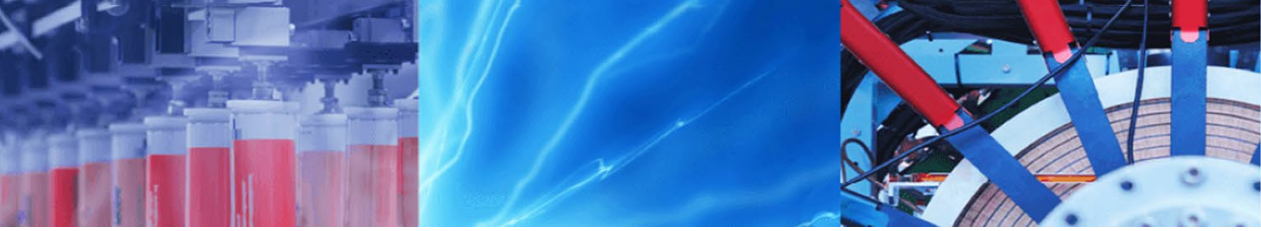

Research Article

\title{
Water flow behaviour in nanochannels: the surface-force effect and slip length
}

\author{
Meng Lu' ${ }^{1}$ (D) Luke D. Connell ${ }^{1} \cdot$ Hongwu Lei ${ }^{2}$
}

(c) Springer Nature Switzerland AG 2019

\begin{abstract}
A set of continuum-based flow equations in nanochannels is developed in terms of the general mass and momentum conservation equations. A local, surface-force-included equation of state is used to replace those conventional, bulk ones employed in traditional hydrodynamics. The flow equations are deduced to a form with three coupled ordinary differential equations, but the mass and momentum conservation laws are rigorously satisfied for long nanochannels where the velocity component vertical to the channel wall is present due to the surface-force effect. The present study demonstrates that: under conditions in which the flowrates are realistically accessible, water flow profiles in nanochannels may greatly deviate from the routine molecular dynamics (MD) simulations. Those predicted profiles by extensive MD studies may only apply to the limiting case in which unrealistically large driving-forces in the streaming direction are applied. Hence, the 'slip length' obtained by MD simulations in this way would not be suited to characterise the pertinent slippage effect and the flow enhancement in many cases commonly encountered in real scenarios. The results obtained in this work allow one to have new insights into liquid flow profiles in nanochannels and can be taken to be a useful reference for possible comparison of MD simulations and laboratory tests.
\end{abstract}

Keywords Nanochannel flow $\cdot$ Surface force $\cdot$ Slip length $\cdot$ Local equation of state $\cdot$ Continuum

\section{Introduction}

A conduit whose characteristic dimension (e.g., the diameter of a round tube or the gap-width of a slit) is below $100 \mathrm{~nm}$ can roughly be defined as a nanochannel, and fluid behaviour there will fall into the regime of nanofluidics (e.g., Eijkel and van den Berg [1] and Schoch et al. [2]). Study of non-wetting liquid behaviour in nanochannels (e.g., carbon nanotubes) has attracted intensive interest over the past two decades [1-4], because some liquids, such as water, may have much higher flowrate in hydrophobic nanotubes than that predicted by the traditional hydrodynamics (e.g., see [5-8]). This unique characteristic may potentially bring a great benefit to a broad range of applications, including in the biological and new energy areas. On the other hand, understanding of wetting or partially wetting liquid behaviour in nanochannels is also of significant importance in many fields. For instance, geological disposal of contaminated wastes in subsurface requires the knowledge of fluid flow in tight rocks, where a significant proportion of pores is on the nanoscale. The pertinent knowledge obtained may help better assess the potential risks environmentally involved. While in the unconventional, geological energy area associated with shale/tight-rock reservoirs, significant resources are stored in nanopores $([9,10])$. Understanding of the pertinent flow/transport mechanisms may significantly help enhanced recovery of resources combined with $\mathrm{CO}_{2}$-sequestration techniques.

The water flow profile in a nanochannel may deviate greatly from that in macroscopic channels. This is because the interaction between the solid wall of the channel and the water molecules, which is trivial on the macroscopic scale, plays a key role in this context. The behaviour of

Meng Lu, meng.lu@csiro.au | ${ }^{1}$ CSIRO Energy, Clayton, Australia. ${ }^{2}$ IRSM, Chinese Academy of Sciences, Wuhan, China.

SN Applied Sciences (2019) 1:1185 | https://doi.org/10.1007/s42452-019-1225-6 
water confined in nanochannels then becomes surfacedominated rather than bulk-dominated, with the combined effect of the two nearby surfaces engaged.

Molecular dynamics (MD) techniques are routinely used in nanofluidics (e.g., [11-15] and the references therein), as they may provide a theoretically consistent methodology in this context, especially when the nanochannel's dimension is below $1 \mathrm{~nm}$, or even approaches the diameter of a water molecule. In these extremely narrow channels, the continuum approach fails. Many interesting water behaviours, such as the single-file water transport scenario (e.g., [16]) and the anomalous water-phase theme near a solid wall (e.g., [17]), occur there with the molecular configuration changed. These phenomena can only be accounted for by computer simulations based on molecular or atomistic considerations.

However, an MD method, in essence, is stochasticthereby may bringing considerable uncertainties into the relevant computations for pressure-driven nanoflow problems. For example, to overcome the thermal noise effect in MD simulations, one needs to set an ultra-large, often unrealistic driving-force (e.g, greater than $10^{10} \mathrm{~g}$, where $g$ denotes the gravity), making the streaming velocity of water on the order of $10^{1} \sim 10^{2} \mathrm{~m} / \mathrm{s}[14,18]$. Such flowrate and driving-force are significantly beyond realistic scenarios. In MD simulations, the slip length, which is traditionally an important parameter to characterise the slippage effect and the flow enhancement in nanoflows (e.g., [19-22]), is determined routinely by extrapolation of those ultra-high flowrates to hypothetically lower ones. Few studies are available currently to discuss the validity of such treatment, but apparently it is at least one of the reasons why the slip length obtained by MD simulations and laboratory tests often scatters so greatly (e.g., [14, 22, 23]). In fact, due to the intrinsic statistical essence in MD simulations for liquid flow in nanochannels, there is even a dispute over the flow profile shape fitted by regression of the results computed-i.e., whether it is the Poiseuille type or plug-like [23].

Here we present a continuum-based analysis to water or liquid flow in some not-extremely-narrow nanochannels for which the continuum theory is applicable. The lower bound of the continuum theory for liquid flow in nanochannels has not definitely defined yet, and many studies based on MD simulations, laboratory tests and theoretical analyses suggest that it would be in the range of above $1 \mathrm{~nm}$ to several nanometres. For example, in the review article [4], Bocquet and Charlaix, after summarising many results based on laboratory tests and MD simulations, concluded that ' $1 \mathrm{~nm}$ ' can be roughly regarded as the lower bound of continuum theory. Below this bound, the continuum concept breaks down, while above it, "the Navier-Stokes (N-S) equations can be safely used to account for the fluid transport". While in another review article, Noy et al. [3] thought that the lower bound of the continuum assumption for nanoflows should be set as $2 \mathrm{~nm}$ and stated that "... flow through CNTs with lateral dimension of less than $2 \mathrm{~nm}$ would fall in the domain of the nascent field of non-continuum fluidics". However, in a later review article [24], Sparreboom et al. recommended, as a rule of thumb, that the lower bound can be set to be 10 molecular size, which is consistent with the results presented by Qiao and Aluru [25]. In a recent research work by Tian et al. [26], the authors again confirmed that 10-molecular-size can be suitably served as the limit for the continuum theory. Therefore, here we suggest that, in practice, the continuum-based nanoflow flow equations proposed in this work can be conservatively used in nanochannels whose width is of 10 molecular size or greater, even though these equations can be formally used in narrower ones in a straightforward way. Nanochannels/ nanopores in such a range are typically seen in tight rocks present in the real geological world (e.g., [9]).

With the continuum analysis the issues encountered in MD simulations can be evaded. The main purpose of the present work is to provide some new insights into the relevant nanoflow profiles that cannot be simulated by the MD methods due to the thermal noise effect. A major contribution of this work against those based on the traditional, bulk hydrodynamics is that: instead of using the conventional, bulk equations of state (EoS), a local, surface-forceincluded EoS [27-30] is used to develop the pertinent flow equations for long nanochannels. These flow equations developed are described by a set of ordinary differential equations, but they comply with the mass and momentum conservation law rigorously when the non-streaming velocity component exists owning to the surface-force effect. Since third-order derivatives of velocities are present in these equations, additional boundary conditions need to be specified. Discussions are presented to address how these unconventional boundary conditions can be supplemented.

Three examples are presented to validate the present flow model; the first two are related with MD simulations and the last one is by a two-dimensional analysis based on the $\mathrm{N}-\mathrm{S}$ equations.

An important observation obtained in this study is that: under realistic flow conditions, the water flow profiles in nanochannels may significantly differ from MD simulations if slippages is allowed on the channel walls. Also, the slipping velocity on the wall can be negative and it is not a constant but position-dependent. Thus, the applicability of slip length becomes invalid in this context. The results demonstrated in this work can be taken as useful references for possible comparison of MD simulations and laboratory tests, as these equations are derived with 
rigorous consideration of conservation laws. This work also motivates us to raise an open question-is the slip length obtained by MD simulations really accurate for realistic nanoflows? If not, how can the slippage effect that relates to the actual flow enhancement be correctly determined/ characterised when the non-streaming velocity component is present?

\section{Nanoflow flow equations with surface-force effect}

Here we consider liquid flow in a two-dimensional (2D) slit with a high length/gap-width aspect ratio (Fig. 1). We let the 2D-channel's length and gap-width be $L$ and $H$, respectively, where $L>>H$. We set the $x$-direction along the channel's length for streaming flow, and the $y$-direction vertical to the channel wall. The two solid surfaces (channel walls) are located at $y=0$ and $y=H$, respectively.

We suppose that the flow is steady and isothermal. The mass and momentum conservation equations for a 2D-plane flow can be described by:

$\partial_{x} \rho u_{x}+\partial_{y} \rho u_{y}=0$,

$$
\begin{aligned}
\rho\left(u_{x} \partial_{x} u_{x}+u_{y} \partial_{y} u_{x}\right)= & -\partial_{x} p+2 \partial_{x}\left(\mu \partial_{x} u_{x}\right) \\
& +\partial_{y}\left[\mu\left(\partial_{x} u_{y}+\partial_{y} u_{x}\right)\right]+\rho f_{x}
\end{aligned}
$$

$$
\begin{aligned}
\rho\left(u_{x} \partial_{x} u_{y}+u_{y} \partial_{y} u_{y}\right)= & -\partial_{y} p+2 \partial_{y}\left(\mu \partial_{y} u_{y}\right) \\
& +\partial_{x}\left[\mu\left(\partial_{y} u_{x}+\partial_{x} u_{y}\right)\right]+\rho f_{y}
\end{aligned}
$$

where $\rho$ is the fluid density, $u_{x}$ and $u_{y}$ are the flow velocities in the $x$ - and $y$-direction, respectively, $p$ is pressure, $\mu$ the (shear) viscosity, and $\rho f_{x}$ and $\rho f_{y}$ denote the body forces exerted by the external fields in the $x$ - and $y$-directions, respectively.

We now let $\partial_{x} p=0$ and $f_{y}=0$, and use $\rho f_{x}$ as the driving-force for streaming flow. Such treatment is consistent with the method used in MD simulations, allowing us to

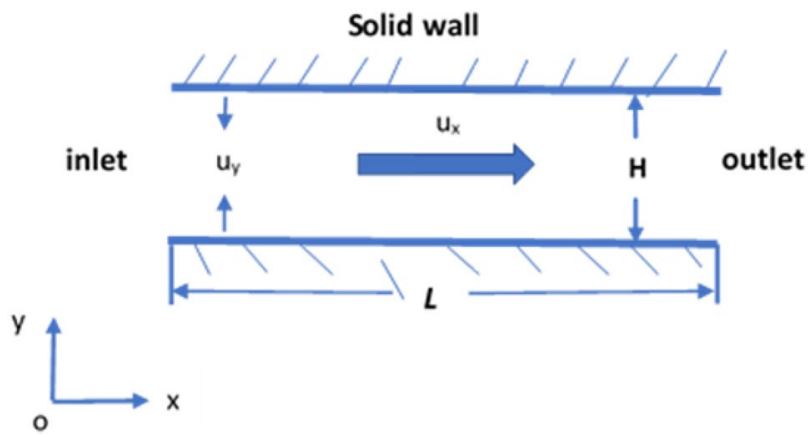

Fig. 1 Schematic of a 2-dimensional plane slit channel directly compare the results obtained here with those by MD simulations.

The local EoS given in Berkeley and Carey [30] is described by:

$p=f_{R K}(\rho, T)+\Phi_{W}(H, y)$

where $f_{R K}(\rho, T)$ represents the standard Redlich-Kwong EoS for bulk fluid, in which $T$ denotes temperature, while

$\Phi_{W}=k_{W}\left\{\left(1-2 D_{f}^{6} / 15 y^{6}\right) / y^{3}-\left[1-2 D_{f}^{6} / 15(y-H)^{6}\right] /(y-H)^{3}\right\}$

describes the additional pressure contribution associated with the intermolecular interaction between the fluid and the two solid walls, in which $k_{W}$ is a parameter proportional to the Hamaker constant $\left(A_{\|}\right)$and can be approximately treated as a constant for water. $D_{f}$ is the diameter of a molecule of the fluid concerned, which for water is approximately equal to $2.8(2.75) \AA$.

This local EoS was formulated following a rigorous procedure of statistical thermodynamics. It treats the water as an uncharged simple fluid and uses the Lennard-Jonestype potential function to account for the long-rangeforce effect present in the region between the two nearby walls as well as that between fluid molecules.

Clearly, Eq. (4) is for a long slit channel without considering the end-effect of the channel, and is fully consistent with the flow case concerned in this study.

Considering that $L>>\partial_{x} p=0$ and $\Phi_{W}$ is only a function of $y$ in Eq. (4), one may approximately take $u_{y}=u_{y}(y)$ and $\rho=\rho(y)$ when neglecting the end-effect. Namely, $u_{y}$ and $\rho$ are functions of y merely, and they are independent of $x$. Thus, for Eq. (1) one has $\partial_{y} \rho u_{y}=\left(\rho u_{y}\right)^{\prime}$ where $(\cdot)^{\prime}=d(\cdot) / d y$. Integration of Eq. (1) with respect to $x$ then gives:

$u_{x}(x, y)=-\rho^{-1}\left(\rho u_{y}\right)^{\prime} \cdot x+u_{x^{\prime}}^{*}$

where $u_{x}^{*}$ is the 'constant' of integration, which is actually a function of $y$.

Equation (6) shows that $u_{x}$, in contrast to its $y$-directional counterpart $u_{y}$, depends on both $x$ and $y$, and that it has two components: one stems from the $y$-directional flow through mass conservation and is (linearly) $x$-dependent, while the other, denoted by $u_{x}^{*}$, is a function of $y$ only.

The streaming mass flowrate $(q)$ can then be evaluated by:

$q=\int_{0}^{H} \rho u_{x} d y=-\left.\left(\rho u_{y}\right)\right|_{y=0} ^{y=H} \cdot x+\int_{0}^{H} \rho u_{x}^{*} d y=0+\int_{0}^{H} \rho u_{x}^{*} d y$,

which is determined by $u_{x}^{*}(y)$ merely and is identical at any $x$-location as $u_{y}(0)=u_{y}(H)=0$ (no-penetration boundary condition). 
It is noted that the mass flowrate $q$ is determined by $u_{x^{\prime}}^{*}$ but $u_{x}^{*}$ is not a directly observable quantity; what can be directly measured in practice is $u_{x}$ rather than $u_{x}^{*}$.

Substitution of Eq. (6) into Eqs. (2) and (3) gives:

$$
\begin{aligned}
& \left\{\mu\left[\rho^{-1}\left(\rho u_{y}\right)^{\prime}\right]^{\prime \prime}-\rho u_{y}\left[\rho^{-1}\left(\rho u_{y}\right)^{\prime}\right]^{\prime}+\rho^{-1}\left(\rho u_{y}\right)^{\prime 2}\right\} \cdot x \\
& +\left[-\mu u_{x}^{* \prime \prime}+\rho u_{y} u_{x}^{* \prime}-\left(\rho u_{y}\right)^{\prime} u_{x}^{*}-\rho f_{x}\right]=0
\end{aligned}
$$

$\mu\left[\rho^{-1}\left(\rho u_{y}\right)^{\prime}\right]^{\prime}-2 \mu u_{y}^{\prime \prime}+\rho u_{y} u_{y}^{\prime}+C_{\rho} \rho^{\prime}+\Phi_{W}^{\prime}=0$,

where $C_{\rho}=\partial_{\rho} f_{R K}(\rho, T)$ denotes the fluid compressibility. For water or supercritical gases under high pressures, one may use the linear approximation of $C_{\rho}$ due to their small compressibility. Namely, $C_{\rho} \approx \partial_{\rho} f_{R K}\left(\rho_{0}, T\right)$ in which $\rho_{0}$ is the fluid density at a static reference state under a reference pressure $p_{0}$. Consequently, the equation of state (4) can be approximately described by

$p-p_{0}=C_{\rho}\left(\rho_{0}, T\right) \cdot\left(\rho-\rho_{0}\right)+\Phi_{W}(H, y)$

Since $x$ in Eq. (8) is an arbitrary value, one must make

$\mu\left[\rho^{-1}\left(\rho u_{y}\right)^{\prime}\right]^{\prime \prime}-\rho u_{y}\left[\rho^{-1}\left(\rho u_{y}\right)^{\prime}\right]^{\prime}+\rho^{-1}\left(\rho u_{y}\right)^{\prime 2}=0$,

and

$\mu u_{x}^{* \prime \prime}-\rho u_{y} u_{x}^{* \prime}+\left(\rho u_{y}\right)^{\prime} u_{x}^{*}+\rho f_{x}=0$

simultaneously to comply with the $x$-directional momentum conservation law.

Equations (9)-(12) provide three coupled ordinary differential equations to determine three variables: $\rho, u_{y}$, and $u_{x}^{*}$. These equations can be equivalently recast into the dimensionless ones. Towards this we let $u_{x}^{0}$ and $u_{y}^{0}$ be two representative values of $u_{x}^{*}$ and $u_{y}$, respectively; let $\bar{y}=y / H$, $\bar{\rho}=\rho / \rho_{0}, \bar{u}_{x}^{*}=u_{x}^{*} / u_{x}^{0}$ and $\bar{u}_{y}=u_{y} / u_{y}^{0}$. Substitution of these dimensionless quantities into Eqs. (9)-(12) yields:

$\left[\bar{\rho}^{-1}\left(\bar{\rho} \bar{u}_{y}\right)^{\prime}\right]^{\prime}-2 \bar{u}_{y}^{\prime \prime}+\lambda \bar{\rho} \bar{u}_{y} \bar{u}_{y}^{\prime}+\kappa_{0} \bar{\rho}^{\prime}+\kappa_{1} \bar{\Phi}_{w}^{\prime}(\bar{y})=0$,

$\left[\bar{\rho}^{-1}\left(\bar{\rho} \bar{u}_{y}\right)^{\prime}\right]^{\prime \prime}-\lambda\left\{\bar{\rho} \bar{u}_{y}\left[\bar{\rho}^{-1}\left(\bar{\rho} \bar{u}_{y}\right)^{\prime}\right]^{\prime}-\bar{\rho}^{-1}\left(\bar{\rho} \bar{u}_{y}\right)^{\prime 2}\right\}=0$,

$\bar{u}_{x}^{* \prime \prime}-\lambda\left[\bar{\rho} \bar{u}_{y} \bar{u}_{x}^{* \prime}-\left(\bar{\rho} \bar{u}_{y}\right)^{\prime} \bar{u}_{x}^{*}\right]+\bar{\rho} \bar{f}_{x}=0$.

Here, $\lambda=\rho_{0} u_{y}^{0} H / \mu$ denotes the relevant Reynolds number in the $y$-direction, while $\kappa_{0}=H \rho_{0} C_{\rho} / \mu u_{y}^{0}$, $\kappa_{1}=H P_{0} / \mu u_{y^{\prime}}^{0} \bar{\Phi}_{W}^{\prime}=\Phi_{W}^{\prime} / P_{0}$, and $\bar{f}_{x}=H^{2} \rho_{0} f_{x} / \mu u_{x}^{0}$.
The natural boundary conditions can be specified as follows:

$\bar{u}_{y}(0)=\bar{u}_{y}(1)=0, \bar{\rho}(0)=\bar{\rho}(1)=\bar{\rho}_{W}$, and $\bar{u}_{x}^{*}(0)=\bar{u}_{x}^{*}(1)=u_{s l i p^{\prime}}^{*}$

in which $\bar{\rho}_{W}=\rho_{W} / \rho_{0}$ while $\rho_{W}$ represents the liquid density on the wall, and $u_{s l i p}^{*}$ is the slipping velocity of the fluid on the boundary; $u_{\text {slip }}^{*}$ can be either zero or non-zero with the slip condition, but must be zero with the no-slip condition, which will be further discussed in the following.

Since Eq. (14) is a third-order differential equation for both $\bar{u}_{y}$ and $\bar{\rho}$, while Eq. (16) only provides four relevant relations on the two boundaries, two more ones are needed and can be separately defined for the non-slip and the slip condition as follows.

For the non-slip condition $\left(\left.u_{x}\right|_{\text {on wall }}=0\right)$, one has:

$\bar{u}_{y}^{\prime}(0)=0$

in view of Eq. (6) as $x$ is an arbitrary value there; another boundary condition can be obtained from Eq. (15) by setting it on the wall, i.e.,

$\bar{u}_{y}^{\prime \prime}(0)-\bar{\rho}^{\prime}(0) \bar{u}_{y}^{\prime}(0) / \bar{\rho}_{W}-\kappa_{0} \bar{\rho}^{\prime}(0)-\kappa_{1} \bar{\Phi}_{W}^{\prime}(0)=0$.

Equation (18) is the requirement of momentum conservation on the boundary, and therefore it is a complex, but accurate boundary condition.

For the slip condition $\left(\left.u_{x}\right|_{\text {on wall }} \neq 0\right)$, Eq. $(17)$ is no longer applicable, and one needs to introduce another boundary condition to replace it. The supplementary one can be obtained by setting Eq. (13) on $\bar{y}=0.5$, which gives

$\bar{u}_{y}^{\prime \prime}(0.5)=0$,

because the flow profile is symmetrical with respect to $\bar{y}=0.5$, and therefore $\bar{u}_{y}(0.5)=\bar{\rho}^{\prime}(0.5)=\bar{\Phi}_{w}^{\prime}(0.5)=0$.

Equations (13)-(15), along with the boundary conditions specified above, define a boundary-value problem. In practice we found that this problem is numerically difficult to solve. Thus, we reduce it to one that is approximate, but more easily solvable.

Considering $\bar{\rho} \approx 1$ and $\bar{\rho}^{\prime}<<1$ for water, we take

$\bar{\rho}^{-1}\left(\bar{\rho} \bar{u}_{y}\right)^{\prime}=\bar{u}_{y}^{\prime}+\bar{u}_{y} \bar{\rho}^{\prime} / \bar{\rho} \approx \bar{u}_{y}^{\prime}$.

The relevant relative error $\left(\varepsilon_{u}\right)$ is:

$\varepsilon_{u}=\left|\bar{u}_{y} \bar{\rho}^{\prime} /\left(\bar{\rho} \bar{u}_{y}\right)^{\prime}\right|$.

We shall estimate $\varepsilon_{u}$ later and demonstrate that (20) is a good approximation for water to the cases of interest. 
Substituting Eq. (20) into Eq. (13) and differentiating the resulting equation over $\bar{y}$ again, one has:

$\bar{u}_{y}^{\prime \prime \prime}-\lambda\left(\bar{\rho} \bar{u}_{y} \bar{u}_{y}^{\prime \prime}+\bar{\rho} \bar{u}_{y}^{\prime 2}+\bar{u}_{y} \bar{u}_{y}^{\prime} \bar{\rho}^{\prime}\right)-\kappa_{0} \bar{\rho}^{\prime \prime}-\kappa_{1} \bar{\Phi}_{w}^{\prime \prime}=0$.

While Eq. (14) becomes

$\bar{u}_{y}^{\prime \prime \prime}-\lambda\left(\bar{\rho} \bar{u}_{y} \bar{u}_{y}^{\prime \prime}-\bar{\rho} \bar{u}_{y}^{\prime 2}\right)=0$.

Equations (19), (22) and (23), along with Eqs. (16)-(18), constitute a new set of nanoflow equations. Note that (19) is no longer needed for these equations and, as can be observed later, it will be automatically satisfied in computations.

The flow equations developed above, unlike the classic Blasius boundary-layer equation, have no self-similar solutions because of the characteristic dimension $\mathrm{H}$ present in these nanoflow equations via Eq. (4) or (10).

In the following computations, the actual boundary conditions are not set exactly on the two walls because $\Phi_{W}$ becomes singular there. Rather, they are set at two locations slightly away from the two walls, with a small distance of $\delta_{W}=0.75 D_{f}$. Then $\bar{\delta}_{W} \equiv \delta_{W} / H=0.75 D_{f} / H$. It can be shown that the impact of the singularity to flow profiles will be stabilised when $y>0.75 D_{f}$.

\section{Results, model validation and discussions}

\subsection{Results with the present model}

Below we discuss some water flow profiles in several typical cases.

Case 1 Static state. This applies to the scenario for a long nanochannel with two dead-ends, within which the water is in a still state at $p_{0}$ and with $\bar{f}_{x}=\bar{u}_{x}=\bar{u}_{y} \equiv 0$. Flow Eqs. (19), (22) and (23) are then simplified to:

$\kappa_{0} \bar{\rho}^{\prime}+\kappa_{1} \bar{\Phi}_{W}^{\prime}=0$.

The pertinent density profile is shown in Fig. 2 , in which the widths of nanochannel are 4, 6 and $8 \mathrm{~nm}$, respectively. Since the water diameter is around $0.275 \mathrm{~nm}$, accordingly the relevant channel's width is approximately of 14,22 and 29 molecular size. The parameters used here and in the following are given in Table 1. The figure shows that the water density in the $y$-direction, even in the static state, is not uniform because of the presence of the surface-force effect. It can be shown that the change of the water density in the $y$-direction, even though it is small (Fig. 2), cannot be completely ignored in the equations; otherwise, Eq. (13) or (22) cannot be hold.

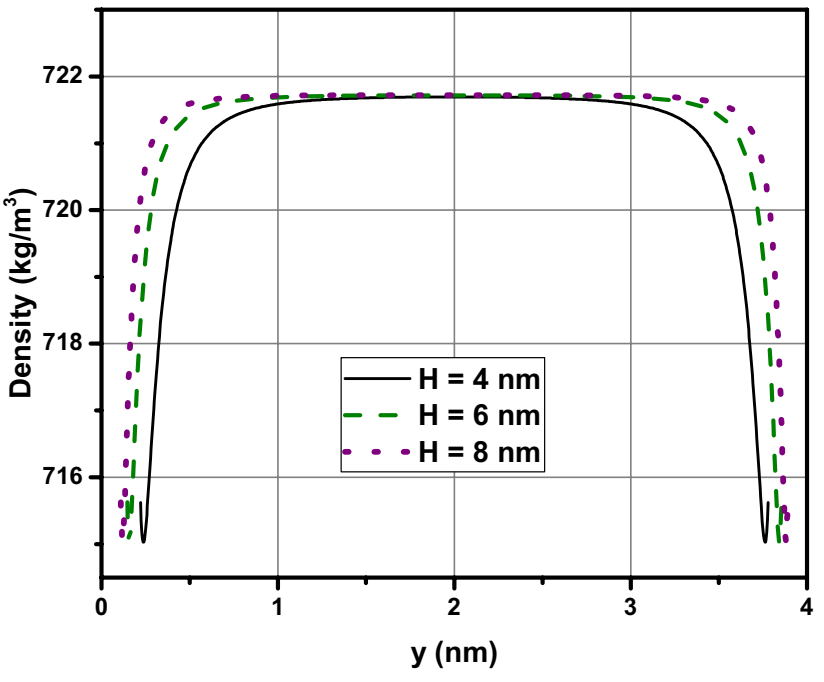

Fig. 2 Static distributions of water density along the y-direction in four channels, with $\mathrm{H}=1.24,2.48,3.72$ and $4.96 \mathrm{~nm}$, respectively

Case 2 Self-conserved flow. This applies to the scenario of a nanochannel that is horizontally placed into a water-bath without the gravity effect, where the two ends of the channel are opened. In this case, $\bar{f}_{x} \equiv 0$ so that $\bar{u}_{x}^{*} \equiv 0$ and $q \equiv 0$ [Eq. (7)], but the total $x$-directional flow (i.e., $u_{x}$ ) may not vanish. While for $\bar{u}_{y}$, it is subject to two conditions-the non-slip condition and the slip condition.

1. Under the non-slip condition, we found that no nontrivial solutions for $\bar{u}_{y}$ can be obtained-namely, $\bar{u}_{y} \equiv 0$, implying that the water in the channel is fully in a static state. The water behaviour then becomes that of Case-1.

2. Under the slip condition, nontrivial $u_{y}$-solution exists, which is plotted in Fig. 3.

As $u_{y} \neq 0$ and $u_{y}^{\prime} \neq 0$ under the slip condition (Fig. 3 ), $u_{x}$ is no longer identical to zero according to Eq. (6) even though $u_{x}^{*} \equiv 0$ now. Figure 4 illustrates a typical flow velocity-vector field of water in the channel of $H=2.75 \mathrm{~nm}(10$ molecular size). This figure reveals two numerical observations:

(i) the $x$-directional flow forms vortex-like patterns in the channel, and it is self-conserved in the sense of mass conservation, i.e., $q \equiv 0$ at any $x$-location; and

(ii) slippage occurs on the wall, and the slipping velocity is negative and $x$-location dependent, but this slippage does not contribute to the total mass flow $q$ along the channel's length.

The pertinent water density profile is almost identical to that shown in Fig. 2, and thus it is not presented here. 
Table 1 Major parameters used in computations

\begin{tabular}{|c|c|c|c|c|c|c|c|c|c|}
\hline $\begin{array}{l}\text { Molecular } \\
\text { diameter }\end{array}$ & $\begin{array}{l}\text { Distance to } \\
\text { wall }\end{array}$ & Temperature & Viscosity $^{a}$ & $\begin{array}{l}\text { Hamaker } \\
\text { constant }\end{array}$ & $\begin{array}{l}\text { Reference } \\
\text { pressure }\end{array}$ & $\begin{array}{l}\text { Reference }^{c} \\
\text { density }\end{array}$ & $\begin{array}{l}\text { Critical }^{\mathrm{a}} \text { pres- } \\
\text { sure }\end{array}$ & $\begin{array}{l}\text { Critical }^{\mathrm{a}} \\
\text { tempera- } \\
\text { ture }\end{array}$ & $\begin{array}{l}\text { Density }{ }^{d} \text { at } \\
\delta_{W}\end{array}$ \\
\hline$D_{f}(n m)$ & $\delta_{W}(n m)$ & $\mathrm{T}(\mathrm{K})$ & $\mu(\mathrm{Pa} s)$ & $A_{\|}(J)$ & $\mathrm{P}_{0}(\mathrm{MPa})$ & $\rho_{0}\left(\mathrm{~kg} / \mathrm{m}^{3}\right)$ & $\mathrm{P}_{\mathrm{C}}(\mathrm{Pa})$ & $\mathrm{T}_{\mathrm{C}}(\mathrm{K})$ & $\rho_{W}\left(\mathrm{~kg} / \mathrm{m}^{3}\right)$ \\
\hline 0.275 & 0.21 & 278.15 & $1.3 \times 10^{-3}$ & $1 \times 10^{-20}$ & 1 & 721.5 & 22 & 647 & 717.9 \\
\hline
\end{tabular}

${ }^{\text {a } D a t a}$ from http://webbook.nist.gov

${ }^{b}$ The Hamaker constant depends on the solid and fluid species of interest, and its value is typically in the range of one faction to a few of $10^{-20}(\mathrm{~J})$ (e.g., see, [30-32])

'Calculated using the standard R-K EoS at $P_{0}$ and $T$ specified

${ }^{\mathrm{d} C}$ Calculated using local EoS Eq. (4) at $\delta_{W}=0.23 \mathrm{~nm}\left(0.75 \mathrm{D}_{\mathrm{f}}\right)$ at $P_{0}$ and $T$

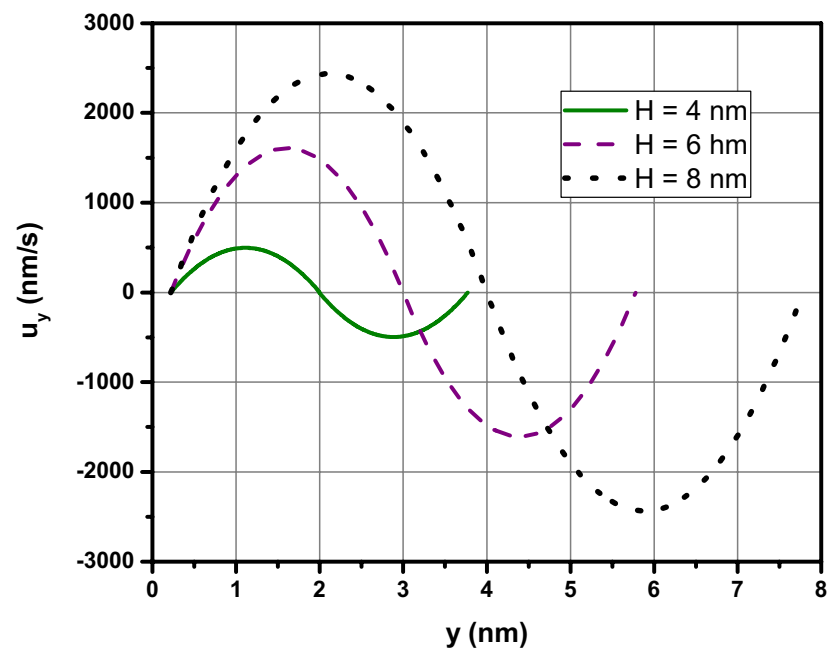

Fig. 3 The $y$-directional velocity profiles in channels with gapwidth of $\mathrm{H}=2,4$ and $6 \mathrm{~nm}$, respectively. No streaming driving-force is applied here

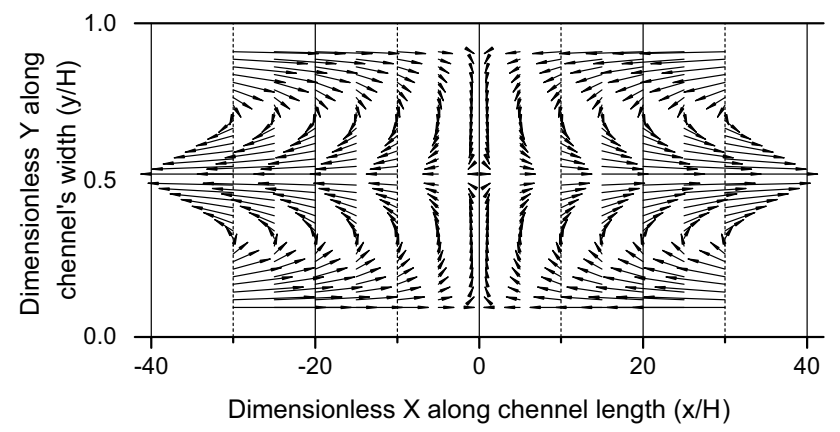

Fig. 4 The flow velocity-vector field of water in the channel of $\mathrm{H}=2.75 \mathrm{~nm}$ (10 water molecular size), where no streaming force is applied. The length of the channel is $\mathrm{L}=80 \mathrm{H}(220 \mathrm{~nm})$

It is noted that the flow pattern illustrated in Fig. 4 is an idealised result without consideration of the energy conservation. Theoretically, without input of external energy to the whole system (the channel + water), the

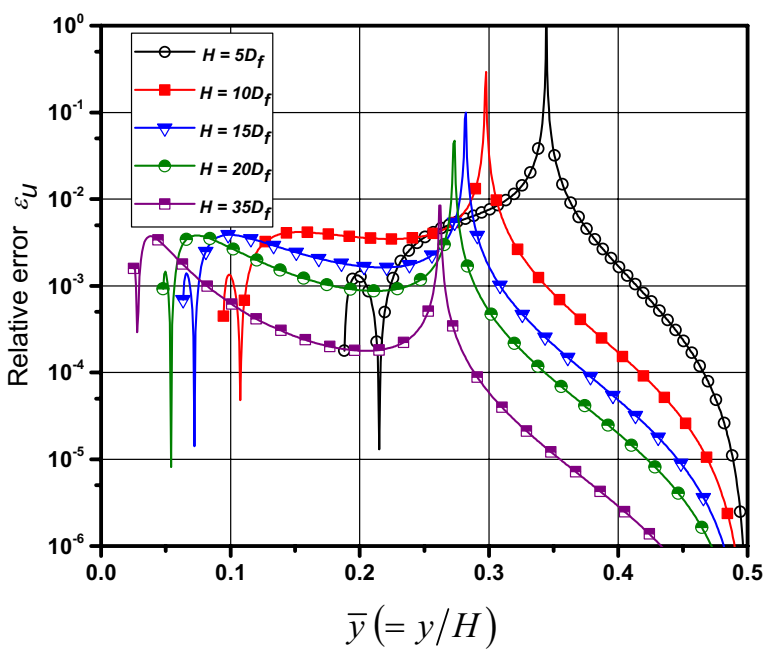

Fig. 5 Relative errors estimated by Eq. (20). The widths of channel concerned are $5,10,15,20$ and $35 D_{f}$, respectively. $D_{f}$ is the molecular diameter which is around $0.275 \mathrm{~nm}$ for wter

isothermal condition cannot be held permanently with energy dissipation of the viscous flow.

Since $\bar{u}_{y}$ is unrelated to $\bar{u}_{x}^{*}$ or $\bar{f}_{x}$ according to Eqs. (22) and (23), we may use the solution obtained above to estimate the errors $\varepsilon_{u}$ (Fig. 5) in terms of Eqs. (20) and (21).

From Fig. 5 one can see that the errors are generally on or below $O\left(10^{-1}\right)$ except at those locations of $\bar{u}_{y}^{\prime}=0$ and their immediate vicinities, where the errors may reach $O(1)$. Also, the errors decrease with the increase of $H$. For example, for $H=10 D_{f}(=2.75 \mathrm{~nm})$, the error is approximately on $O\left(10^{-2}\right)$, while for $H=35 D_{f}(\sim 9.6 \mathrm{~nm})$, the error is dropped to around $O\left(10^{-3}\right)$.

Case-3 Body-force-driven streaming flow. In this case $\bar{f}_{x} \neq 0$ so that $\bar{u}_{x}^{*} \neq 0$. The flow profiles are also subject to two boundary conditions - the non-slip condition and the slip condition.

\section{SN Applied Sciences}


1. Non-slip condition. Under this condition no nontrivial solutions for $u_{y}$ can be found. Namely, $u_{y} \equiv 0$ and then $u_{x} \equiv u_{x}^{*}$.Thus, the $u_{x}$-profile is described by

$\bar{u}_{x}^{\prime \prime}+\bar{\rho} \bar{f}_{x}=0$.

The water density profiles are identical to those shown in Fig. 2.

Since the water density is nearly a constant (Fig. 2), the $\bar{u}_{x}$-profiles described by Eq. (25) are approximately the Poiseuille type. Thus, we can arrive at an interesting conclusion: the body-force-driven streaming flow profile of water in a nanochannel tends to be the Poiseuille type if no slippage occurs on the wall, regardless of whether the surface-force effect is considered.

2. Slip condition. In this case, the boundary condition specified by Eq. (17) is no longer applicable. Instead, we use Eq. (18) to replace it. Since $u_{\text {slip }}^{*}$ can be either zero or non-zero in this case, in the following we discuss the relevant flow profiles separately.

(i) When $u_{\text {slip }}^{*}=0$, Fig. 6 shows the relevant $u_{x}$-profiles at four $x$-positions along the channel's length $(x=0,10,20$ and $30 \mathrm{H})$ in the channel of $H=4 \mathrm{~nm}$, with $f_{x}=1.4 \times 10^{5} \mathrm{~g}$ (approximately equivalent to $\partial_{x} p=1000 \mathrm{MPa} / \mathrm{m}$ as the drivingforce). This figure shows that in this case the effect of the first term of Eq. (6) is significantly greater than its second counterpart, and the $\Lambda^{-}$ shape of $u_{x}$-profile is formed. Like the $u_{x}^{*}$-profile (at $x=0$ ) there, the maximum $u_{x}$ is also at the centre of the channel, but negative slipping velocities occur on the channel's boundary.

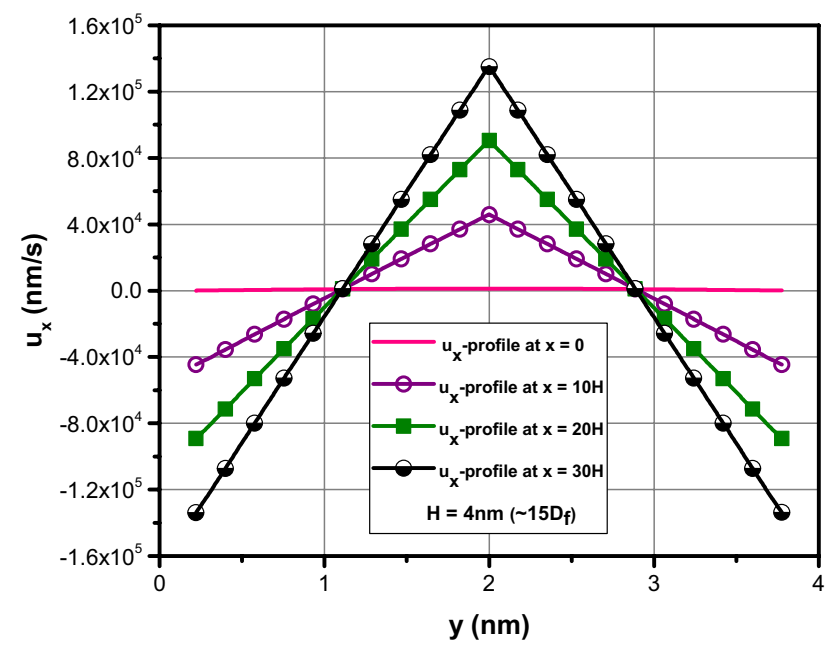

Fig. 6 The $u_{x}$-profiles of water flow in a channel of $\mathrm{H}=4 \mathrm{~nm}$ at $\mathrm{x}=0$, 10,20 and $30 \mathrm{H}$, respectively; the body force applied is $\mathrm{f}_{\mathrm{x}}=1.4 \times 10^{5} \mathrm{~g}$ (equivalent to the pressure gradient of $10^{9} \mathrm{~Pa} / \mathrm{m}$ in the $\mathrm{x}$-direction)
However, as demonstrated in (7), these negative, $x$-dependent slipping velocities have no contributions to the streaming mass flowrate $q$. .It can be shown that the flow velocity-vector field corresponding to Fig. 6 is similar to that of the right half part of Fig. 4 (the inlet is now set at $x=0$ ); however the flow is no longer self-conserved as $q \neq 0$ here.

(ii) When the driving-force is increased to $f_{x}=1.4 \times 10^{7} \mathrm{~g}$ (Fig. 7), the effect of the second term in Eq. (6) overwhelms its first counterpart. Then, the $u_{x}$-profile in this case approaches that of $u_{x}^{*}$. If the driving-force is further increased to the level that MD simulations usually use (greater than $10^{10} \mathrm{~g}$ ), the effect of the $y$-directional flow on the streaming flow can be completely ignored, and the streaming flow would be overwhelmingly dominated by the $u_{x}^{*}$-profile. In that case, the $u_{x}$-profile would literally become a Poiseuille flow with small negative slipping velocities on the wall.

(iii) When $u_{\text {slip }}^{*} \neq 0$, in this case the $u_{y}$-profiles will not be altered because they are not associated with $u_{\text {slip }}^{*}$. While the relevant $u_{x}^{*}$-profile is simply a superimposition of that obtained under $u_{\text {slip }}^{*}=0$ plus the non-zero $u_{\text {slip }}^{*}$. Clearly, when $u_{\text {slip }}^{*}$ is sufficiently large, the slipping velocity of water on the wall would become positive. Then, the $u_{x}$ -profile would tend to be plug-like (a Poiseuille flow plus a positive slipping velocity) with extremely large driving-forces.

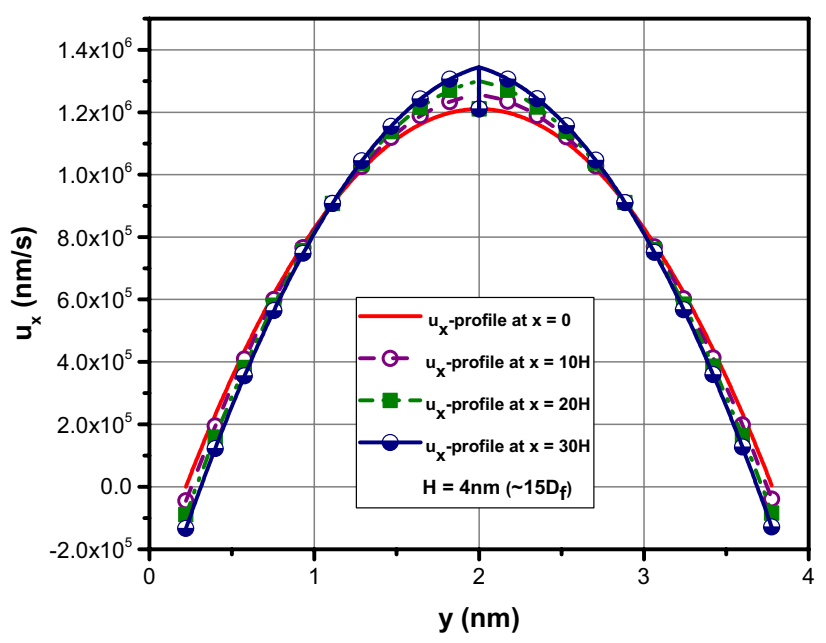

Fig. 7 The $u_{x}$-profiles of water flow in a channel of $\mathrm{H}=4 \mathrm{~nm}$ at the distance of $x=0,10,20$ and $30 \mathrm{H}$, respectively; the external load exerted is $f_{x}=1.4 \times 10^{7} \mathrm{~g}$ (equivalent to the pressure gradient of $10^{11}$ $\mathrm{Pa} / \mathrm{m}$ in the $\mathrm{x}$-direction) 


\subsection{Model validation}

We provide three examples to validate the flow model proposed in this paper. The first two present the comparison of the result obtained by the present model with the pertinent $M D$ simulation, in which ultra-large driving forces are applied. The last one concerns a case in which a realistic, much smaller external force is applied. In this case the relevant MD simulation data, to the best of our knowledge, are not available for such pressure-driven flow problems. Therefore, we conduct a 2D analysis to compare and discuss the result obtained by the present model.

\section{Example 1 Flow profile in a nanochannel}

Figure 8 demonstrates the comparison of the result by the present method and that by the MD simulation given in Kannam et al. [18], in which the driving force of $10^{14}$ $\mathrm{Pa} / \mathrm{m}$ (equivalent to the acceleration of $10^{11} \mathrm{~m} / \mathrm{s}^{2}$ used in [18]) is applied. In our computation (Fig. 8), the slipping velocity $u_{\text {slip }}^{*}=14.75 \mathrm{~m} / \mathrm{s}$ is used, which is very close to $14.9 \mathrm{~m} / \mathrm{s}$ of the slip velocity obtained in [18]. It is not surprising that a good agreement is obtained by the present method and the MD simulation in this case, because, as addressed previously, with such a large, unrealistic driving force in the streaming direction, the $y$-directional molecular movements can be ignored, and the flow profile can be described by the Poiseuille flow plus a constant slip velocity.

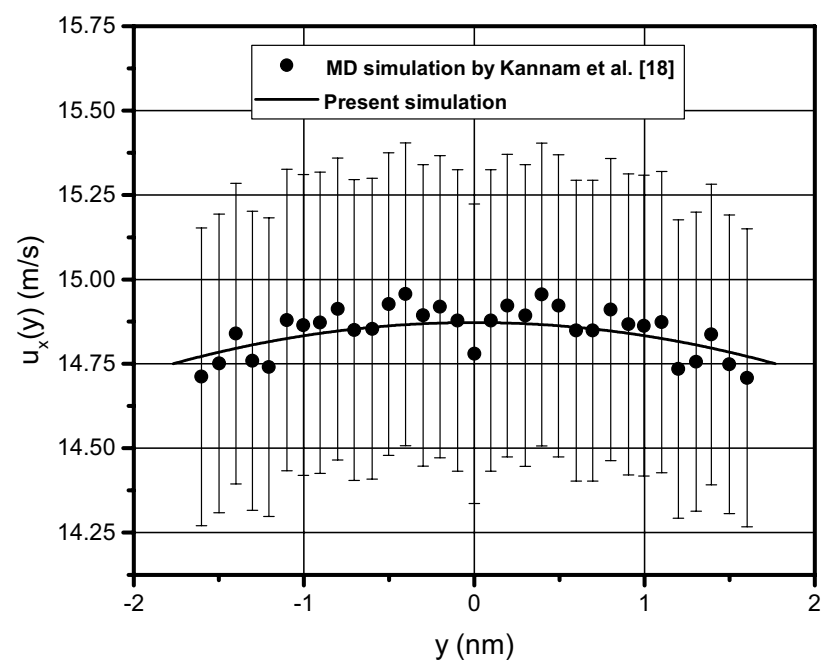

Fig. 8 Comparison of the result by the present method and that by the MD simulation presented in Kannam et al. [18]. The width of channel is $4 \mathrm{~nm}$, and the exerted external force (acceleration) is $10^{11} \mathrm{~m} / \mathrm{s}^{2}$ in [18], which is equivalent to $10^{14} \mathrm{~Pa} / \mathrm{m}$ of the pressure gradient in the streaming direction. The error bars shown in the figure are associated with the MD simulation
Example 2 Average flowrates in nanochannels

Figure 9 demonstrates the average velocities in nanoslits with different widths and under different pressure gradients [33]. Both the MD simulations given in [33] and the present model predictions are illustrated in this figure. Note that the fluid species concerned is methane rather than the water. Hence, the molecular diameter is now $D_{f}=0.41 \mathrm{~nm}$. According to the source literature [33], the viscosity of the fluid varies with the width of the nanochannel, and we use the pertinent values in our computations shown in Fig. 9.

From Fig. 9 one can see that the computational results obtained by the present model agree well with the pertinent MD simulations. Interestingly, even for the nanochannel with $H=7 D_{f}$ (which is less than $10 D_{f}$ ) the predictions by the continuum-based model still reasonably well agree with that by the MD simulation.

It is noted that in [33] Zhang et al. did not explicitly specify what the slip length or the slipping velocity was obtained with their simulations, except for the case of $H=12 D_{f}$ in which the slipping velocity of around $20 \mathrm{~m} / \mathrm{s}$ was observed. However, in our simulations the non-slip boundary conditions are used thoroughly, implying that no slipping velocity is needed to match the pertinent MD simulations. Hence, we tend to suggest that the slipping velocity obtained by Zhang et al. was caused by the regression error instead of being a physically correct value.

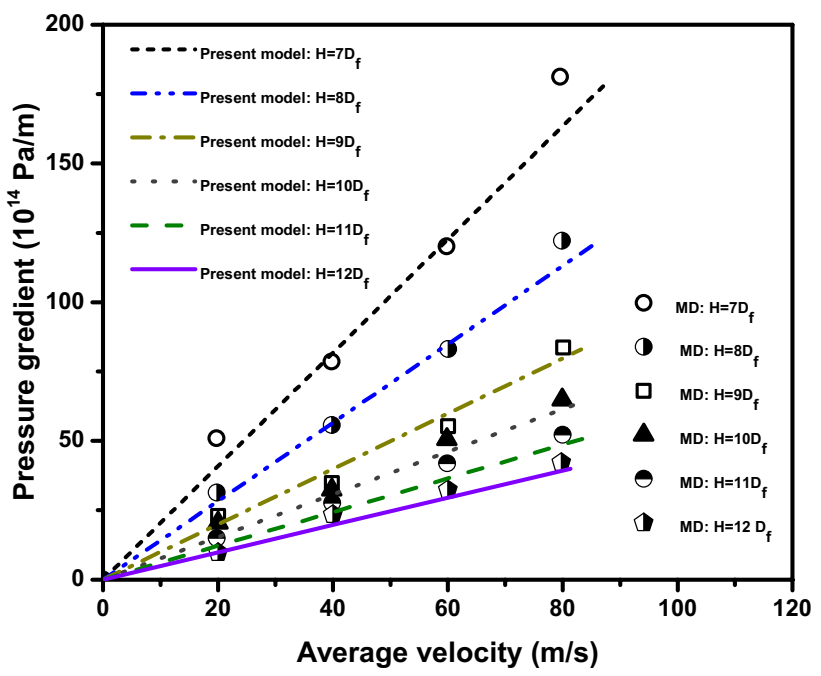

Fig. 9 Simulation results for the average flow velocity of methane in nanochannels under different external loads. According to the source literature [33], the viscosities of the fluid are approximately $1.03 \times 10^{-4}, 0.97 \times 10^{-4}, 0.95 \times 10^{-4}, 0.94 \times 10^{-4}, 0.93 \times 10^{-4}$ and $0.91 \times 10^{-4} \mathrm{~Pa} \cdot \mathrm{s}$ for $\mathrm{H}=7,8,9,10,11$ and $12 \mathrm{D}_{\mathrm{f}}$, respectively. For methane, $D_{f}=0.41 \mathrm{~nm}$, and its critical pressure and temperature are 4.59 $\mathrm{MPa}$ and $190.6 \mathrm{~K}$, respectively 
Example 3 Verification by 2D model

We now consider the flow profile of water in a nanochannel with a small driving force (pressure gradient) of $5 \times 10^{5} \mathrm{~Pa} / \mathrm{m}$, which greatly contrasts the driving force typically used in MD simulations (e.g., $\sim 10^{14} \mathrm{~Pa} / \mathrm{m}$, as shown in Examples 1 and 2). Such pressure gradient can be typically seen in reality. Other parameters used here are the same as those listed in Table 1 . Since no relevant MD simulations are available to compare, we conduct a two-dimensional simulation to verify the present model in this context.

The 2D slit geometry is set with $\mathrm{H}=4 \mathrm{~nm}$ and $\mathrm{L}=20 \mathrm{~nm}$ (Fig. 1). The inlet is at $x=0$ and the outlet is at $x=L$. The solid boundaries are set at $y=0$ and $y=H$, respectively. We use the commercial ComSol Multiphysics Modeling software (https://www.comsol.com) to carry out the relevant $2 \mathrm{D}$ analysis by solving the $\mathrm{N}-\mathrm{S}$ equations described by Eqs. (1)-(3). The user-defined fluid property function in ComSol is used, with which the local EoS expressed by Eq. (10) is employed to describe the relationship between pressure and density.

Again, we discuss two cases here; one is for the non-slip boundary condition and the other for the slip condition at the two solid walls (Fig. 1).

With the non-slip boundary condition, the 2D flow problem described by Eqs. (1)-(3) along with Eq. (10) is well-posed. Figure 10 presents the computational results for the $x$-directional velocity by ComSol and the present model, respectively. It can be seen from this figure that, under the non-slip boundary condition, the result obtained by the present model agrees quite well with that by the $2 D$ analysis even in the near-end region. While for the $y$-directional velocity of the fluid in the channel, the $2 \mathrm{D}$ analysis by ComSol also predicts that its magnitude is virtually zero. This confirms the conclusion that we draw in Case-3, Point-1 of the last sub-section, which is "under this condition no nontrivial solutions for $u_{y}$ can be found. Namely, $u_{y} \equiv 0$ and then $u_{x} \equiv u_{x}^{* \prime \prime}$.

With the slip boundary condition, the 2D flow problem described by Eqs. (1)-(3) becomes ill-posed. It can be shown that in this case ComSol only generates nonphysical solutions with its built-in slip boundary condition in ComSol. In contrast, the present model, as discussed in the last sub-section, permits one to solve the relevant flow equations and provides useful new insights/understandings to the relevant velocity profiles when slippage is allowed on the channel's wall and non-streaming velocity (y-direction) components are present in the channel.

\subsection{Additional notes and remarks}

Several notes pertaining to the results presented above follow.

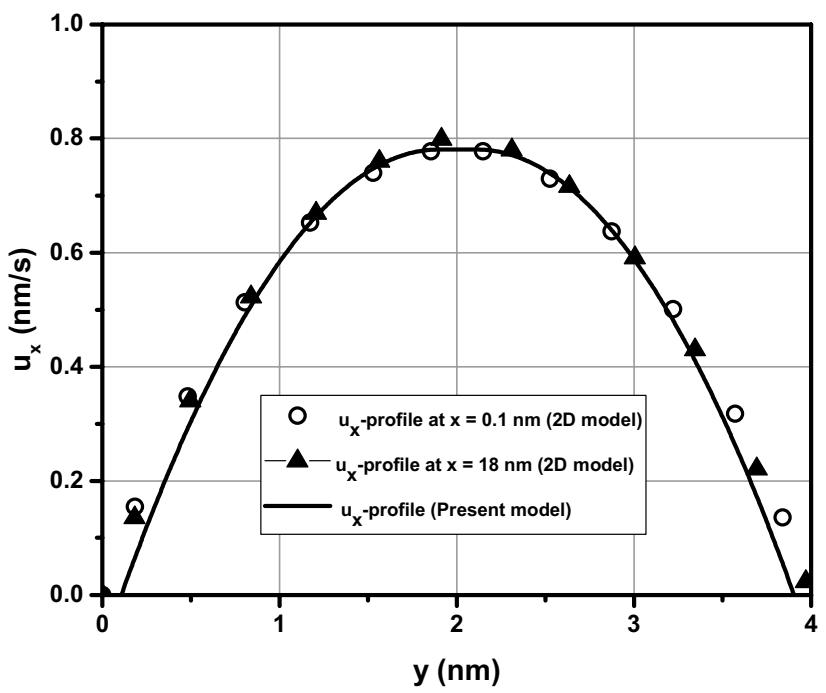

Fig. 10 Comparison of the 2D simulation and the present model for water flow in a nanochannel with $\mathrm{H}=4 \mathrm{~nm}$ and $\mathrm{L}=20 \mathrm{~nm}$. The pressure gredient is $0.5 \mathrm{MPa}$. The $\mathrm{u}_{\mathrm{x}}$-profiles by the $2 \mathrm{D}$ simulation at $x=0.1 \mathrm{~nm}$ (immediately close to the inlet of the channel) and $x=18 \mathrm{~nm}$ (close to the outlet of the channel) are shown in the figure. The the result by the present model is also shown there

1. These flow patterns presented in Figs. 4, 6 and 10 may not be replicated by routine MD simulations, because the flowrates studied here are significantly below the thermal noise usually encountered in MD simulations.

2. The $u_{y}$-profiles are sensitively dependent on $\Phi_{W}$ that was derived in terms of the Lennard-Jones-type potential function. If different intermolecular potential functions are used, $\Phi_{W}$ would be different, and one can expect that some details of $u_{y}$-profiles would be altered accordingly. For example, the $y$-directional velocity may change its direction, subject to that the surface force involved is attractive or repulsive [34].

3. The local EoS developed by Berkeley and Carey [30] treated the water as an uncharged fluid, and other forces-e.g., the electrostatic force (e.g., see [1, 2, 4, 34, 35] etc.) - are not considered there. Hence, the present analysis based on this EoS cannot describe the flow details in relation to any deletion or adsorption layers caused by those forces. However, extension of the present approach to accommodate these effects is straight forward, provided that they can be incorporated into a local EoS such as Eq. (4).

4. We use the bulk viscosity (Figs. $2,3,4,5,6,7,8$ ) or the local bulk viscosity (Fig. 9) in the computations. Some experiments (e.g., [6]) and MD simulations (e.g., $[15,33]$ and the references therein) showed that $\mu$ is size-dependent in nanochannels, and its value may remarkably be greater or smaller than its bulk counterpart. Logically, when the surface-force takes 
a role in nanochannels, the viscosity should also be $y$-position-dependent rather than a constant. If a position-dependent viscosity along with the non-slip boundary condition is used, the nanoflow equation problem described by Eqs. (1)-(3) is well-posed, and the flow enhancement, if any, can be fully determined/ predicted without introducing additional parameters such as the slipping velocity and slip length. This deserves further investigations both in theory and experiment.

\section{Conclusions}

1. When the non-slip condition applies, the water flow profile in a nanochannel is approximately the Poiseuille type, regardless of whether the surface-force effect is considered.

2. When slippage is allowed to occur on the wall, the water flow profiles may significantly deviate from the MD predictions in realistic flow conditions, in which the molecular movements normal to the wall may become comparable with or even greater than their counterparts along the channel's length. Thus, position-dependent, vortex-like flow patterns can be formed, and

3. in this context, the parameter slip length would no longer be suited to characterise the slippage effect and the flow enhancement.

4. Those flow profiles in nanochannels predicted by many MD simulations may only apply to the limiting case where an unrealistically large body force is applied.

5. The flow equations developed can be readily extended to including more sophisticated surface-force effects (e.g., the electrostatic force and structural force, etc.), provided that these effects can be incorporated into a local EoS either theoretically or empirically.

\section{Compliance with ethical standards}

Conflict of interest On behalf of all authors, the corresponding author states that there is no conflict of interest.

\section{References}

1. Eijkel JCT, van den Berg A (2005) Nanofluids: what is it and what can we expect from it? Microfluid Nanofluid 1:249-267

2. Schoch RB, Han JY, Renaud P (2008) Transport in nanofluidics. Rev Mod Phys 80:839-883
3. Noy A et al (2007) Nanofluidics in nanotubes. Nanotoday 2(6):22-29

4. Bocquet L, Charlaix E (2010) Naofluidics, from bulk to interface. Chem Soc Rev 39:1073-1095

5. Hummer G, Rasaiah JC, Noworyta JP (2001) Water conduction through the hydrophobic channel of a carbon nanotube. Nature 414(8):188-190

6. Majumder $\mathrm{M}$ et al (2005) Enhanced flow in carbon nanotubes. Nature 438(15):44

7. Holt JK et al (2006) Fast mass transport through sub-2-nanometer carbon nanotubes. Science 312:1034-1037

8. Chen $X$ et al (2008) Nanoscale fluid transport: size and rate effect. Nano Lett 8(9):2988-2992

9. Loucks RG et al (2012) Spectrum of pore types and networks in mudrocks and a descriptive classification for matrix-related mudrock pores. AAPG Bull 96:1071-1098

10. Lu M et al (2017) A coupled, non-isothermal gas shale flow model: application to evaluation of gas-in-place in shale with core samples. J Pet Sci Eng 158:361-379

11. Janeček J, Netz RR (2007) Interfacial water at hydrophobic and hydrophilic surfaces: depletion versus adsorption. Langmuir 23:8417

12. Cao B-Y et al (2009) Molecular momentum transport in fluid-solid interface in MEMS/NEMS: a review. Int J Mol Sci 10:4638-4706

13. Ye HF et al (2011) Size and temperature effects on the viscosity of water inside carbon nanotubes. Nano Res Lett 6:87

14. Kannam SK et al (2013) How fast does water flow in carbon nanotubes? J Chem Phys 138:094701

15. Shaat M (2107) Viscosity of water interfaces with hydrophobic nanopores: application to water flow in carbon nanotubes. Langmuir 33(44):12814-12819

16. Berezhkovskii A, Hummer G (2002) Single-file transport of water molecules through a nanotube. Phys Rev Lett 89(6):064503

17. Leoni F, Franzese G (2016) Effect of confinement between attractive and repulsive walls on the thermodynamics of an anomalous fluid. Phys Rev E 94:062604

18. Kannam SK et al (2012) Slip length of water on graphene: limitations of non-equilibrium molecular dynamics simulations. $J$ Chem Phys 136:024705

19. Barrat J-L, Bocquet $L$ (1999) Large slip effect at a non-wetting fluid-solid interface. Phys Rev Lett 82(23):4671-4674

20. Zhu YX, Granick S (2001) Viscosity of interfacial water. Phys Rev Lett 87(9):096104

21. Cottin-Bizonne et al (2005) Boundary slip on smooth hydrophobic surfaces: intrinsic effects and possible artifacts. Phys Rev Lett 94:056102

22. Huang DM et al (2008) Water slippage versus contact angle: a quasiuniversal relationship. Phys Rev Lett 101:226101

23. Hanasaki I, Nakatani A (2006) Flow structure of water in carbon nanotubes: poiseuille type or plug-like? J Chem Phys $124: 144708$

24. Sparreboom W, van der Buer A, Eijkel JCT (2010) Transport in nanofluidic systems, a review of theory ad applications. New J Phys 12:015004

25. Qiao R, Aluru NR (2003) Ion concentration and velocity profiles in nanochannel electroosmotic flows. J Chem Phys 118(110):4692-4701

26. Tian HH, Huang WZ, Li M, Wang MR (2017) Critical size of continuum theory applicability for single-phase liquid flow in nanochannel. J Nanosci Nanotechnol 17(9):6149-6158

27. Gerweck V, Yadiaroglu G (1992) A local equation of state for a fluid in the presence of a wall and its application to rewetting. Int J Heat Mass Transf 45(7):1823-1832 
28. Carey VP (1999) Statistical thermodynamics and microscale thermophysics. Cambridge University Press, Cambridge

29. Carey VP, Wemhoff AP (2005) Thermodynamic analysis of near-wall effects on phase stability and homogeneous nucleation during rapid surface heating. Int J Heat Mass Transf 48:5431-5445

30. Berkeley P, Carey VP (2013) Thermodynamic analysis of wall effects on phase stability and homogenous nucleation in nanochannels containing superheated liquid. Nanoscale Microscale Thermophys Eng 17:281-303

31. Bergstrőm L (1997) Hamaker constants of inorganic materials. Adv Colloid Interface Sci 70:125-169

32. Takahashi S, Kovscek AR (2010) Wettability estimation of lowpermeability, siliceous shale using surface forces. J Pet Sci Eng 75:33-43
33. Zhang ZQ, Zhang HW, Ye HF (2009) Pressure-driven flow in parallel-plate nanochannels. Appl Phys Lett 95:154101

34. Dergjaguin BV, Churaev NV, Muller VM (1987) Surface forces. Springer, New York

35. Sendner $C$ et al (2009) Interfacial water at hydrophobic and hydrophilic surfaces: slip, viscosity, and diffusion. Langmuir 25(18):10768-10781

Publisher's Note Springer Nature remains neutral with regard to jurisdictional claims in published maps and institutional affiliations. 